\title{
Effect of Humidity on the Permeability of Alcohols in Hydroxylpropyl Xylan Films
}

\author{
Fatemeh Bayati ${ }^{1}$, Yaman Boluk ${ }^{2}$, Phillip Choi ${ }^{1}$ \\ ${ }^{1}$ Department of Chemical and Materials Engineering, University of Alberta \\ Edmonton, Alberta, Canada, T6G 1H9 \\ ${ }^{2}$ Department of Civil and Environmental Engineering, University of Alberta \\ Edmonton, Alberta, Canada, T6G 1H9
}

\section{Extended Abstract}

Inverse gas chromatography (IGC) was used to study the effect of humidity on the permeability of selected low molecular weight alcohols (methanol, ethanol, propanol and butanol) through films made up of a hydrophilic polymer hydroxypropyl xylan (HPX). The thin films used consisted of a neat HPX film and a HPX film with sorbitol, a commonly used food grade plasticizer, (HPX/Sorbitol) and a HPX film with sorbitol and cellulose nanocrystal (CNC) (HPX/Sorbitol/CNC). Diffusivity and solubility of the alcohols at infinite dilution were measured at relative humidity $(\mathrm{RH})$ values of $0 \%, 30 \%, 50 \%$ and $70 \%$ of the carrier gas (helium). Permeability was then calculated using the measured diffusivity and solubility. Diffusion coefficients of the alcohols showed a maximum at about 50\% RH in all films. And solubility coefficients of the alcohols were more or less insensitive to the RH. Permeability coefficients of the alcohols exhibited a maximum at around $50 \% \mathrm{RH}$ as well suggesting that diffusion, not dissolution, dominated the permeability behaviour. The RH dependence of diffusion, thereby permeability, was attributed to the combined effects of plasticizing, swelling, water clustering and alcohol-water clustering. The measured weight-fraction-based Henry's constants suggested that water contents in the films were relatively low, suggesting that water clustering might only take place in the neat HPX film at $70 \% \mathrm{RH}$. 